\title{
Aplicación del método de la madurez para la estimación del plazo de descimbrado de forjados construidos con sistemas industrializados
}

\author{
Application of the maturity method for the estimation of shore removal \\ times in concrete floor slabs built with industrial systems
}

M. C. Castro-Garrido ${ }^{(*)}$, M. C. López-Garzón ${ }^{(*)}, \underline{\text { Y. A. Alvarado }}{ }^{(*)}$, J. O. Castaño $^{(*)},{\text { I. } \text { Gasch }^{(*)}}^{(*)}$

\section{RESUMEN}

El presente artículo propone una metodología para la estimación del plazo de descimbrado de forjados construidos con sistemas industrializados. Para esto, se realiza un estudio de madurez de hormigones usados en este tipo de construcción. Con el fin de construir la curva de madurez en laboratorio se implementa la metodología descrita en la ASTM C1074, calculando la energía de activación por los tres métodos propuestos y midiendo el historial térmico de las mezclas a una temperatura controlada. Por último, se realiza la instrumentación en obra de una placa donde se estima la edad de descimbrado.

Se ha observado que el tiempo de fraguado final no es un parámetro recomendable para descimbrar las placas, como lo recomiendan las productoras de hormigón en Colombia. La edad de descimbrado obtenida es mayor a la del tiempo de fraguado final, habiendo una diferencia de hasta 11 horas en el caso más crítico.

Palabras clave: Madurez; descimbrado; energía de activación; hormigón; sistemas industrializados; tiempos de fraguado.

\section{ABSTRACT}

This article proposes a methodology for estimating the striking time for reinforced concrete slab floors build with industrial systems. For this, a study of maturity of concrete used in such construction is performed. To make the maturity curve in the laboratory, the methodology described in ASTM C1074 is implemented by calculating the activation energy for the three proposed methods and measuring the thermal history of the concrete at a controlled temperature. Finally, a reinforced concrete slab floor is monitored and the striking time is estimated.

It has been observed that the final setting time is not recommended for striking slabs floors, as recommended by the manufacturers of concrete in Colombia. Striking time obtained is higher than the final setting time, having a difference of up to 11 hours at the most critical case.

Keywords: Maturity method; shoring removal; activation energy; concrete; industrial systems; setting time.

(*) Pontificia Universidad Javeriana. Bogotá (Colombia).

${ }^{(* *)}$ ICITECH - Universitat Politècnica de València (España).

Persona de contacto/Corresponding author: alvarado.y@javeriana.edu.co (Y. A. Alvarado)

Cómo citar este artículo/Citation: Castro-Garrido, M. C., López-Garzón, M. C., Alvarado, Y. A., Castaño, J. O., Gasch, I. (2016). Aplicación del método de la madurez para la estimación del plazo de descimbrado de forjados construidos con sistemas industrializados. Informes de la Construcción, 68(541): e131, doi: http://dx.doi.org/10.3989/ic.14.105.

Licencia / License: Salvo indicación contraria, todos los contenidos de la edición electrónica de Informes de la Construcción se distribuyen bajo una licencia de uso y distribución Creative Commons Reconocimiento no Comercial 3.o. España (cc-by-nc). 


\section{INTRODUCCIÓN}

El sistema de construcción en hormigón industrializado, se basa en un sistema estructural conformado únicamente por muros y placas de hormigón (véase Figura 1). Este sistema se utiliza con frecuencia en Colombia, ya que permite acelerar los procesos constructivos de forma económica y práctica (1). El empleo de dichos sistemas se basa en optimizar los tiempos de uso del sistema de encofrado mediante el descimbrado de la estructura en el menor tiempo posible. Esto se debe realizar teniendo en cuenta la edad en la que el hormigón alcanza la resistencia para soportar las cargas que se generan durante el proceso constructivo (2) (3). Este tipo de construcciones resulta ampliamente usado en la construcción de viviendas de interés social o prioritario, gracias a la mejora de plazos y de economía.

El estudio de dichas cargas, generadas durante el proceso constructivo, ha sido abordado por diversos autores. En 1963, Grundy y Kabaila (4), propusieron un método simplificado, que aún se emplea en la actualidad, para determinar las cargas durante la construcción. Este método es de fácil aplicación y está, en la mayoría de los casos, del lado de la seguridad. Más recientemente, investigadores como Duan y Chen (5), Fang et al. (6) y Calderón et al. (7) han desarrollado modelos teóricos simplificados que permiten estimar las cargas entre los puntales y los forjados durante el proceso constructivo de una forma más precisa.

Por otro lado, la medición de la resistencia es importante dado que determina los plazos del descimbrado, para este fin, se debe contar con equipos calibrados. Esto es factible en sis-

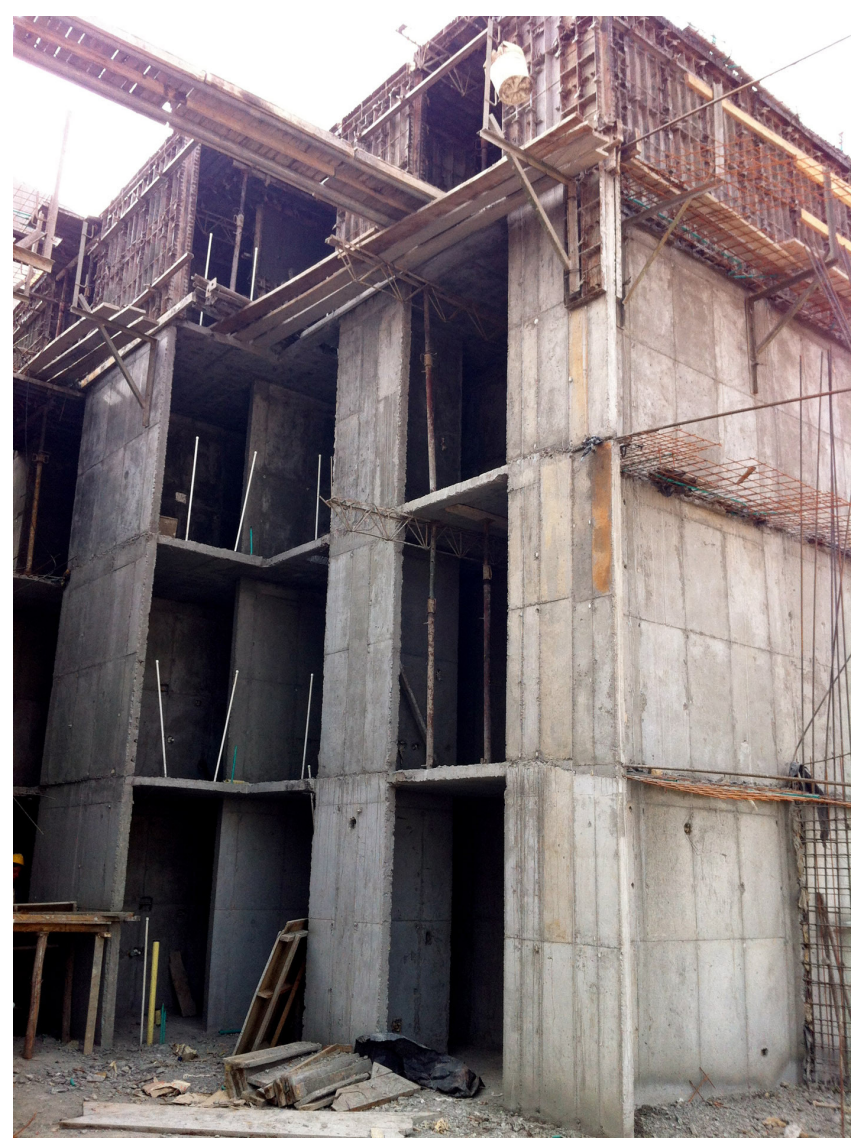

Figura 1. Sistema industrializado. temas constructivos con hormigones convencionales; ya que el tiempo de retiro del encofrado es aproximadamente a los 14 días y desapuntalamiento a los 28 días, por esta razón, los cilindros tomados en obra pueden ser enviados al laboratorio $\mathrm{y}$ de esta forma tener control sobre la resistencia.

Contrario a esto, en sistemas constructivos en los cuales se utilizan hormigones industrializados, los tiempos de retiro del encofrado no van más allá de las 24 horas incluso 12 horas, haciendo que el método para medir las resistencias sea inviable; pues se debería contar con los equipos en la obra. Por esta razón, la decisión de quitar dicho encofrado se hace en base a la experiencia del constructor y a la ficha técnica del hormigón, la cual contiene los tiempos de fraguado final, que es la edad que recomiendan los productores de hormigón para descimbrar (8). Esto ocurre debido a que las normas técnicas no dan parámetros explícitos en cuanto a los plazos para descimbrar la estructura (3).

Por lo anterior, surge la necesidad de implementar un método, que con menor incertidumbre, permita a los constructores tener herramientas técnicas para tomar decisiones en cuanto al descimbrado y a la programación de las obras, teniendo en cuenta que la rotación del sistema de encofrado se podría hacer de una forma más eficiente. Esto se puede lograr mediante la implementación del método de la madurez (9), el cual se ha utilizado en las últimas décadas de forma exitosa para la estimación de la resistencia in situ del hormigón, basándose en su comportamiento térmico (10). Este método, tiene menor incertidumbre ya que la determinación de la curva de madurez, para el hormigón que se utiliza, se realiza en condiciones controladas de laboratorio, por lo que en obra sólo es necesario medir el desarrollo de la temperatura de la mezcla en el elemento. Esto hace que el uso de este método resulte más fiable, dado que hay menos parámetros que afecten los resultados; además es sencillo, porque no requiere mucho tiempo ni personal especializado y resulta ser económico ya que el equipo de medición de temperatura es de fácil acceso para los constructores.

El objetivo principal del presente trabajo es, por tanto, determinar tiempos de fraguado y resistencias a edades tempranas de hormigones industrializados por medio del método de la madurez con el fin de conocer la edad de descimbrado.

\section{PLANTEAMIENTO DEL MODELO EXPERIMENTAL}

Con el objeto de aplicar el método de la madurez para la estimación de resistencias a edades tempranas, en hormigones industrializados, se planteó un estudio experimental en el que se realizaron pruebas de laboratorio y pruebas en obra.

Para el presente estudio se utilizaron dos tipos de mezclas de hormigones industrializados, una mezcla para muro y otra para placa. Estos hormigones son:

- Outinord Muro Cemex - $28 \mathrm{MPa}$

- Outinord Placa Cemex - $21 \mathrm{MPa}$

En ambos casos se utilizaron dos tipos de aditivos: uno para acelerar la resistencia a edades tempranas y otro que actúa como reductor de agua, dándole alta manejabilidad al terminado de las mezclas. 


\subsection{Ensayos realizados}

Para la caracterización de los hormigones empleados en obra se realizaron:

- Ensayos en estado fresco

- Ensayo de asentamiento (ASTM C143-12) (11)

- Ensayo de tiempos de fraguado (ASTM C403-08) (12)

- Ensayos en estado endurecido

- Ensayos de resistencia a compresión en probetas cilíndricas de hormigón (ASTM C39) (13)

- Ensayo de tracción indirecta en probetas cilíndricas de hormigón (ASTM C496-11) (14)

- Ensayo de flexión en probetas prismáticas de hormigón (ASTM C78-10) (15)

- Ensayo para la obtención del módulo de elasticidad en probetas cilíndricas de hormigón (ASTM C469-10) (16)

\subsection{Determinación de la energía de activación y de la curva de madurez}

Para aplicar el método de la madurez, planteado en la ASTM C1074-11 (17), se desarrolló un procedimiento experimental que se dividió en dos partes. La primera se refiere a la determinación de la energía de activación, donde se hicieron ensayos de resistencia a compresión en probetas cúbicas de mortero equivalente, según la norma ASTM C109 (18), y se midieron los tiempos de fraguado de dichos morteros de acuerdo a la norma ASTM C403-08 (12).

En la segunda parte del planteamiento experimental, se midió el historial de temperatura, bajo condiciones controladas, en probetas cilíndricas de hormigón para construir la curva de madurez. Estas probetas se elaboraron con el mismo hormigón usado para los ensayos de resistencia a compresión, tracción y módulo.

\subsection{Calibración del método de madurez}

Buscando validar el método de la madurez implementado en el laboratorio, se realizaron mediciones en hormigón puesto en obra bajo condiciones no controladas. Se elaboraron pro- betas cilíndricas con el hormigón fabricado en la planta de una de las principales productoras de hormigón en Colombia, para una obra situada en Bogotá (Colombia), donde se construye con hormigones con la misma dosificación que los del caso de estudio en el laboratorio.

Se elaboraron 7 probetas cilíndricas para cada mezcla. De las cuales 6 fueron ensayadas a compresión a las edades de 1 , 2 y 7 días. La probeta restante fue usada para determinar el historial de temperaturas. Esto se hizo introduciendo un termopar en el centro de la probeta cilíndrica, el cual estaba conectado a un sistema de adquisición de datos, que registraba valores de temperatura cada 10 segundos (véase Figura 2a). El registro de temperaturas se hizo por un periodo de 7 días (véase Figura 2b).

\section{RESULTADOS}

A continuación se muestran los resultados obtenidos de los diferentes ensayos efectuados.

\subsection{Ensayos de tiempos de fraguado del hormigón}

Los tiempos de fraguado para el hormigón se obtienen tomando mediciones en la cámara de curado a $23{ }^{\circ} \mathrm{C}$ y humedad relativa del $100 \%$. Los resultados obtenidos, tanto por el método gráfico como por el método logarítmico, se muestran en la Tabla 1.

Para los dos hormigones de estudio los valores de tiempos de fraguado inicial y final arrojados por los dos métodos son similares. Por lo tanto, en este caso, se decidió usar los resultados de tiempos de fraguado del método gráfico.

Tabla 1. Tiempos de fraguado de hormigón.

\begin{tabular}{|l|l|c|c|}
\cline { 3 - 4 } \multicolumn{2}{c|}{} & $\begin{array}{c}\text { Método } \\
\text { Gráfico }\end{array}$ & $\begin{array}{c}\text { Método } \\
\text { Logarítmico }\end{array}$ \\
\hline \multirow{2}{*}{ Muro } & Fraguado Inicial (h) & 3,67 & 3,65 \\
\cline { 2 - 4 } & Fraguado Final (h) & 5,50 & 5,61 \\
\hline \multirow{2}{*}{ Placa } & Fraguado Inicial (h) & 2,96 & 2,82 \\
\cline { 2 - 4 } & Fraguado Final (h) & 4,56 & 4,65 \\
\hline
\end{tabular}

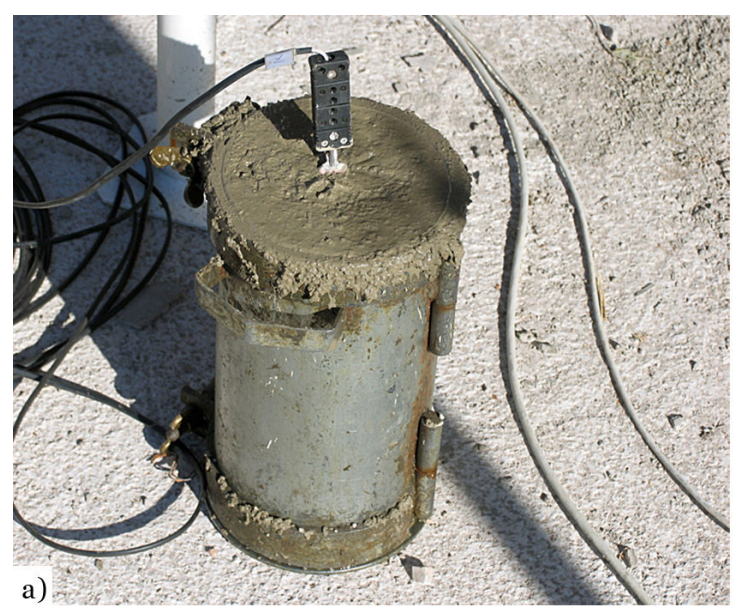

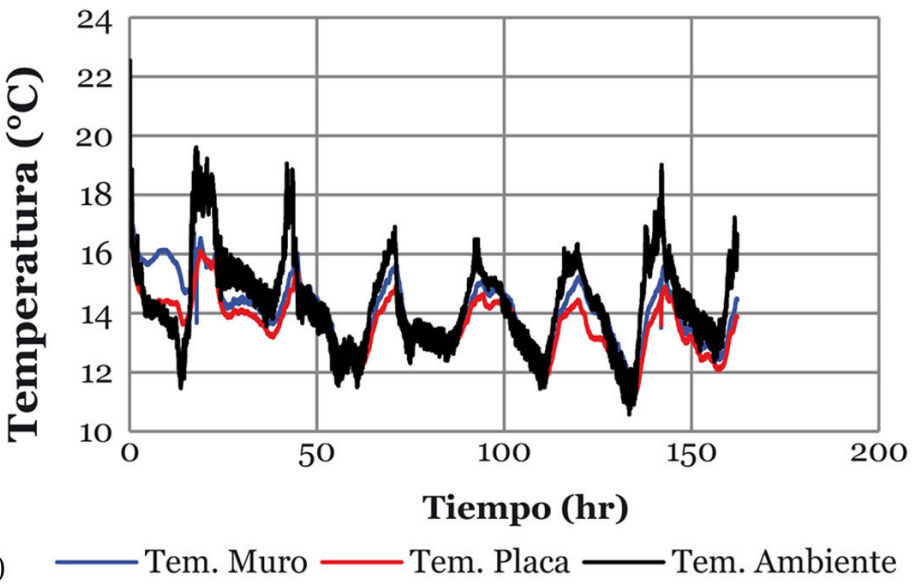

Figura 2. (a) Probeta de concreto instrumentada. (b) Evolución térmica de cilindros curados en condiciones de obra durante 7 días. 


\subsection{Ensayos del hormigón en estado endurecido}

A continuación se muestran los resultados obtenidos de los ensayos practicados al hormigón en estado endurecido, con el fin de determinar la evolución de sus propiedades mecánicas. En la Tabla 2 se pueden observar los resultados de los ensayos realizados.

\subsection{Determinación de la energía de activación}

Siguiendo el procedimiento descrito en la ASTM C1074-11 (17) se determinó el valor de la constante de velocidad $(k)$ para cada uno de los métodos propuestos por la norma, y a partir de dicha constante se obtuvo el valor de la energía de activación (19). En la Tabla 3 se muestran los resultados de energía de activación para la placa y el muro para cada uno de los métodos.

En investigaciones anteriores acerca del tema, se ha demostrado que la energía de activación es única para cada mezcla de hormigón (20), sin embargo la ASTM C107411 (17) sugiere un rango de valor de energía de activación entre 40000 a $45000 \mathrm{~J} / \mathrm{mol}$ para hormigones elaborados con cemento tipo I sin aditivos y/o adiciones, para otras condiciones se sugiere determinar el valor de $Q$ experimentalmente.

En el caso de estudio, las energías de activación calculadas para cada método están en un rango menor al propuesto por la norma ASTM C1074-11 (17). Esto se debe a que este valor es planteado para mezclas que no contienen aditivos ni adiciones.

Por lo tanto, estas energías de activación bajas indican que el hormigón tendrá una edad equivalente mayor en menor tiempo, comparada con una edad equivalente calculada con una energía de activación mayor. Adicionalmente, una energía de activación baja indica que la velocidad de hidratación se va a presentar en menor tiempo.

\subsection{Determinación de la curva de madurez}

Para determinar la edad equivalente, según la ecuación [1], reflejada en la norma ASTM C1074-11 (17), se usa la energía de activación seleccionada y la temperatura de referencia de $23^{\circ} \mathrm{C}$. Esta edad equivalente se calcula para cada intervalo de temperatura.

$$
t=\sum e^{-Q\left(\frac{1}{T_{a}}-\frac{1}{T_{s}}\right)} \Delta t
$$

Donde:

$t$ : Edad equivalente

$$
Q=\frac{E}{R}
$$

Siendo:

$E$ : Energía de activación $(\mathrm{kJ} / \mathrm{mol})$

$R$ : Constante universal de los gases $(8,3144 \mathrm{~J} / \mathrm{K} \mathrm{mol})$

$T_{a}$ : Media de la temperatura durante el intervalo $\Delta \mathrm{t}(\mathrm{K})$

$T_{s}$ : Temperatura de referencia (K)

$\Delta \mathrm{t}$ : Intervalo de tiempo (h)

A partir de las resistencias a compresión obtenidas (véase Tablas 3 y 4) y la edad equivalente se construyeron las curvas de madurez para los dos tipos de hormigón en estudio, siguiendo el procedimiento descrito en el apartado 8 de la norma ASTM C1074-11 (17). La relación que se obtuvo entre el índice de madurez y la resistencia se puede expresar por medio de las siguientes ecuaciones:

Para la placa:

$$
f_{c}^{\prime}=10.608 \cdot L O G(t)-1,1416
$$

Para el muro:

$$
f_{c}^{\prime}=13.674 \cdot \operatorname{LOG}(t)-1,5347
$$

Donde

$f_{c}^{\prime}$ : Resistencia a compresión (MPa)

$t$ : Edad equivalente (horas)

Tabla 2. Resumen propiedades mecánicas. Placa y Muro.

\begin{tabular}{|c|c|c|c|c|c|c|c|c|}
\hline \multirow{2}{*}{ Edad } & \multicolumn{2}{|c|}{$\begin{array}{c}\text { Resistencia Compresión } \\
\text { (MPa) }\end{array}$} & \multicolumn{2}{c|}{$\begin{array}{c}\text { Resistencia Tracción } \\
\text { Indirecta (MPa) }\end{array}$} & \multicolumn{2}{c|}{$\begin{array}{c}\text { Resistencia Flexo } \\
\text { Tracción (MPa) }\end{array}$} & \multicolumn{2}{c|}{$\begin{array}{c}\text { Módulo de Elasticidad } \\
\text { (MPa) }\end{array}$} \\
\cline { 2 - 10 } & Placa & Muro & Placa & Muro & Placa & Muro & Placa & Muro \\
\hline 28 & 29,9 & 38,8 & 2,6 & 3,2 & 4,1 & 5,13 & 22208 & 25035 \\
\hline 14 & 26,1 & 33,7 & 2,3 & 2,7 & 3,9 & 5,05 & 18561 & 23592 \\
\hline 7 & 23,0 & 29,1 & 2,0 & 2,3 & 3,6 & 4,48 & 17406 & 21490 \\
\hline 3 & 18,7 & 23,2 & 1,7 & 1,9 & 3,3 & 3,81 & 15601 & 15946 \\
\hline 1 & 11,5 & 15,3 & 1,3 & 1,5 & 2,3 & 3,46 & 12845 & 15340 \\
\hline
\end{tabular}

Tabla 3. Energía de activación.

\begin{tabular}{|l|c|c|}
\cline { 2 - 3 } \multicolumn{1}{c|}{} & $\begin{array}{c}\mathbf{E}_{\text {placa }} \\
\text { (J/mol) }\end{array}$ & $\begin{array}{c}\mathbf{E}_{\text {muro }} \\
\text { (J/mol) }\end{array}$ \\
\hline Método 1 & 38701,9 & 35254,7 \\
\hline Método 2 & 24811,0 & 32322,2 \\
\hline Método 3 & 31820,0 & 27529,0 \\
\hline
\end{tabular}

Tabla 4. Resistencia a compresión de las probetas cilíndricas de obra.

\begin{tabular}{|c|c|c|}
\hline $\begin{array}{c}\text { Edad } \\
\text { (días) }\end{array}$ & $\begin{array}{c}\text { Resistencia Placa } \\
\text { (MPa) }\end{array}$ & $\begin{array}{c}\text { Resistencia Muro } \\
\text { (MPa) }\end{array}$ \\
\hline 7 & 19,6 & 26,6 \\
\hline 2 & 14,7 & 20,5 \\
\hline 1 & 10,6 & 14,8 \\
\hline
\end{tabular}




\subsection{Calibración del método de madurez}

Los resultados de los ensayos de compresión realizados a las probetas cilíndricas de obra se muestran en la Tabla 4.

Con los perfiles térmicos se calculó la edad equivalente para cada una de las edades de ensayo. Esto se hizo con cada una de las energías de activación y así ajustar la curva de madurez. Posteriormente, se determinó la resistencia a compresión con cada una de las energías de activación de la Tabla 3 y se comparó con la resistencia obtenida en laboratorio como se muestra en la Figura 3.

Se escoge la energía de activación determinada por la constaste de velocidad del primer método, debido a que éste es el que arroja un error menor en cuanto a la resistencias a edades tempranas (cercano al $8 \%$ ) las cuales son las más determinantes en los sistemas industrializados. Además éste es el único que tiene en cuenta los tiempos de fraguado, definiendo una edad inicial de ensayo.

\section{APLICACIÓN CASO DE ESTUDIO}

A partir de los resultados obtenidos del estudio experimental, se realizó un estudio en obra en el cual se pretendía determinar en qué momento se debería retirar el encofrado de una placa de $10 \mathrm{~cm}$ de espesor de hormigón, garantizando que ésta sea capaz de soportar las cargas de construcción. Para ello se instrumentó dicha placa para medir temperaturas directamente sobre el elemento.

Inicialmente se determinó de forma teórica la resistencia del hormigón necesaria para el retiro del encofrado, de acuerdo con el método propuesto por Calavera (9).

Posteriormente, se determinó la edad equivalente en el tiempo de la placa, con base a las temperaturas medidas directamente sobre el elemento.

Por último, conociendo la edad equivalente correspondiente al tiempo de fraguado final del hormigón a una temperatura controlada, se verificó cuál era el tiempo de fraguado en obra. Adicionalmente, usando la ecuación que viene dada por la ASTM C1074-11 (17) se determinó la edad equivalente a la cual se puede garantizar que el hormigón tiene la resistencia para soportar la carga de construcción.

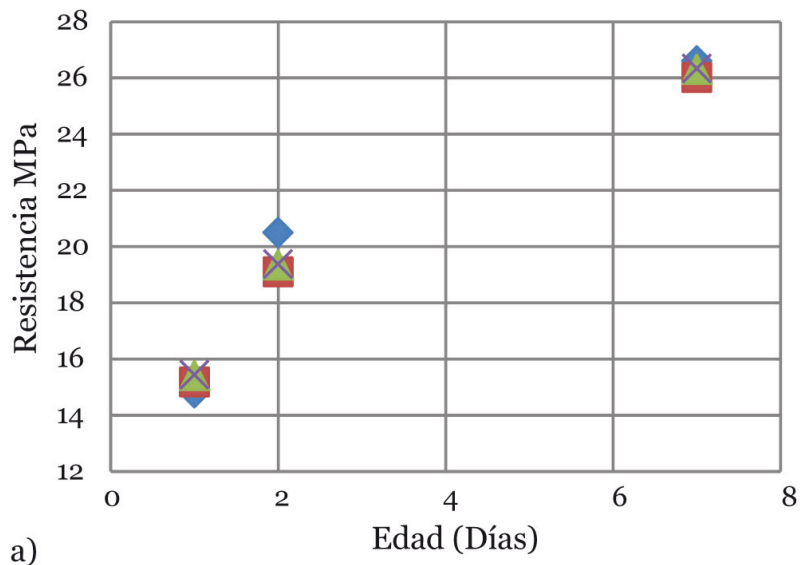

\subsection{Instrumentación del elemento de hormigón}

La instrumentación para la medición de temperatura sobre el elemento de hormigón se llevó a cabo en una obra situada en Bogotá (Colombia) en la cual el hormigón es suministrado por una de las principales productoras de hormigón en Colombia y tiene el mismo diseño de mezcla que el hormigón estudiado en el laboratorio.

Para la instrumentación de la placa se llegó antes de su vaciado, y se verificó que el asentamiento del hormigón estuviera en los rangos de especificación. Una vez vibrado y enrasado se procedió a la instrumentación en diferentes puntos y profundidades de la placa.

La instrumentación se realizó en siete puntos de la placa. Ésta se llevó a cabo con dos instrumentos diferentes de adquisición de datos de temperatura: un madurímetro y un $d a-$ talogger, los cuales estaban conectados a termopares tipo K, que a su vez estaban embebidos en el hormigón por medio de un pitillo, que evita la pérdida del termopar (véase Figura 4). Los equipos midieron la evolución de temperaturas por un periodo de 20 horas aproximadamente, periodo en el cual se considera que el hormigón adquiere la resistencia para retirar el encofrado.

\subsection{Determinación de la resistencia de descimbrado}

Según el método propuesto por Calavera se comparó si la evolución de la placa (Tabla 5) presenta la misma evolución que se muestra en Calavera (21).

En la Tabla 5 se observa que aparte de la evolución de resistencia a compresión, la evolución de resistencia más crítica es la resistencia a tracción indirecta. Por lo cual este método es aplicable a este caso.

Calavera (9) propone que si la carga actuante sobre un forjado al descimbrar es $\beta$.P siendo $P$ la carga total característica del proyecto, (peso propio más cargas permanentes más sobrecargas), el forjado podrá ser descimbrado cuando el hormigón del propio forjado, presente una resistencia a tracción $f_{c t, j}$ tal que:

$$
f_{c t, j} \geq \beta \cdot f_{c t, 28}
$$

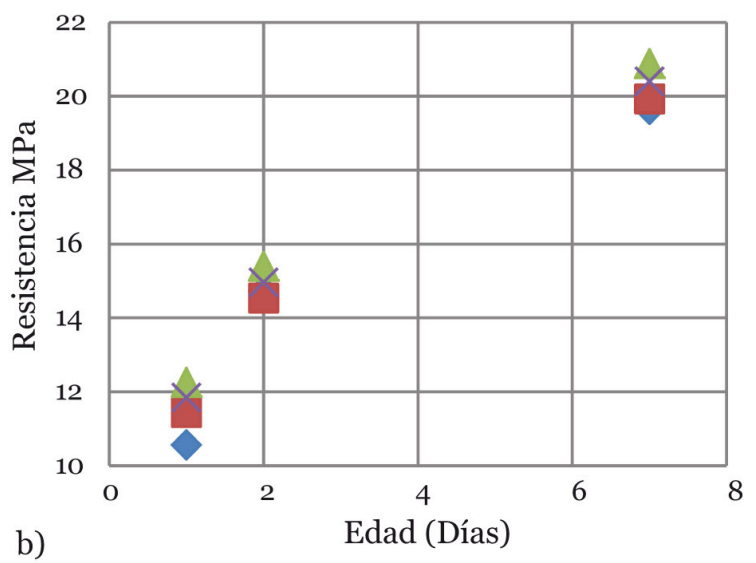

b)

Laboratorio $\square$ Método $1 \triangle$ Método $2 \times$ Método 3

Figura 3. Resistencia calculada con la energía de activación. (a) Muro y (b) Placa. 

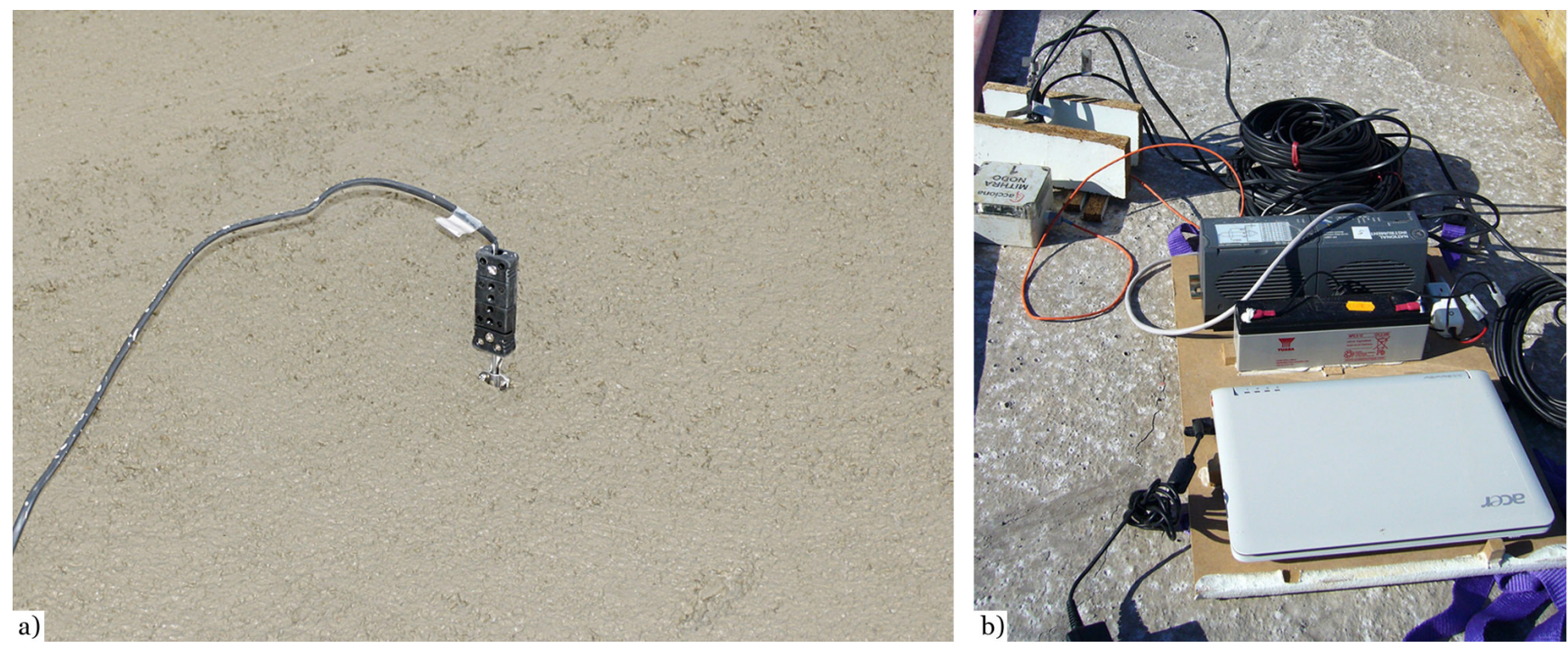

Figura 4. Instrumentación placa edificio industrializado en Bogotá. (a) Placa y (b) Equipos de adquisición de datos y sensores.

Tabla 5. Relación de resistencias en función de la resistencia a compresión del hormigón.

\begin{tabular}{|c|c|c|c|c|}
\hline Edad & $\begin{array}{c}\text { Resistencia Compresión } \\
\text { (\% Evolución) }\end{array}$ & $\begin{array}{c}\text { Resistencia Tracción } \\
\text { Indirecta } \\
\text { (\% Evolución) }\end{array}$ & $\begin{array}{c}\text { Resistencia Flexo } \\
\text { Tracción } \\
\text { (\% Evolución) }\end{array}$ & $\begin{array}{c}\text { Módulo de Elasticidad } \\
\text { (\% Evolución) }\end{array}$ \\
\hline 28 & 100 & 100 & 100 & 100 \\
\hline 14 & 87 & 87 & 94 & 84 \\
\hline 7 & 77 & 75 & 89 & 78 \\
\hline 3 & 63 & 64 & 80 & 70 \\
\hline 1 & 38 & 51 & 56 & 58 \\
\hline
\end{tabular}

Siendo:

$f_{c t, j}:$ Resistencia a tracción en un tiempo $j(\mathrm{MPa})$

$f_{c t, 28}:$ Resistencia a tracción de diseño (MPa)

$$
\beta=\frac{D_{C} \cdot F_{S 1}^{\prime}+L_{C} \cdot F_{S 2}^{\prime}}{D \cdot F_{S 1}+L \cdot F_{S 2}}
$$

Donde

$D_{c}$ : Cargas muertas en el proceso de construcción $(\mathrm{kN})$

$D$ : Cargas muertas de diseño $(\mathrm{kN})$

$L_{c}$ : Cargas vivas en el proceso de construcción $(\mathrm{kN})$

$L$ : Cargas vivas de diseño $(\mathrm{kN})$

Fs: Factor de mayoración de diseño

F's: Factor de mayoración para las cargas de construcción

En las Tablas 6 y 7 se presentan las cargas calculadas para el caso de estudio. Éstas se evaluaron para la etapa de construcción y de diseño.

En cuanto a los factores de mayoración, se tomó un factor de mayoración de 1 para las Cargas de Construcción puesto que ni en la NSR-10 y ni en la ACI 347-04 se consideran factores de mayoración para este tipo de cargas.

Para las Cargas de Diseño, se adoptan los valores especificados en la NSR-10 (22), sección B.2.4.2, siendo éstos de 1,6 para las Cargas Muertas y 1,2 para las Cargas Vivas.
Tabla 6. Evaluación de cargas de construcción.

\begin{tabular}{|l|l|c|}
\hline \multicolumn{2}{|c|}{ Tipo de Carga } & $\begin{array}{c}\text { Carga } \\
\left(\mathbf{k N} / \mathbf{m}^{\mathbf{2}}\right)\end{array}$ \\
\hline \multirow{4}{*}{ Muertas } & Encofrado & 0,18 \\
\cline { 2 - 3 } & Hormigón & 2,40 \\
\cline { 2 - 3 } & Total & 2,58 \\
\hline \multirow{4}{*}{ Vivas } & Trabajadores & 0,88 \\
\cline { 2 - 3 } & Equipos & 0,30 \\
\cline { 2 - 3 } & Materiales & 0,10 \\
\cline { 2 - 3 } & Total & 1,28 \\
\hline
\end{tabular}

Tabla 7. Evaluación de cargas de diseño.

\begin{tabular}{|l|l|c|}
\hline \multicolumn{2}{|c|}{ Tipo de Carga } & $\begin{array}{c}\text { Carga } \\
\left(\mathbf{k N} / \mathbf{m}^{\mathbf{2}}\right)\end{array}$ \\
\hline \multirow{4}{*}{ Muertas } & Peso Propio & 2,40 \\
\cline { 2 - 3 } & Baldosa cerámica & 0,80 \\
\cline { 2 - 3 } & Pañete de Yeso & 0,25 \\
\cline { 2 - 3 } & Total & 3,45 \\
\hline \multirow{3}{*}{ Vivas } & Uso Residencial & 1,80 \\
\cline { 2 - 3 } & Total & 1,80 \\
\hline
\end{tabular}


Aplicando la ecuación [6] y sustituyendo en la ecuación [5], se obtiene:

$$
f_{c t, j} \geq 0,643 \cdot f_{c t, 28}
$$

Puesto que la resistencia que se toma usualmente en obra es la resistencia a compresión, se deseaba encontrar una relación entre la resistencia a tracción y la resistencia a compresión, para el tipo de hormigón en estudio. Esta relación se determina a partir de los datos obtenidos en el laboratorio. Según Calavera (21) la relación entre las resistencias a tracción y compresión tiene una tendencia representada por la siguiente ecuación potencial:

$$
f_{c t} \geq \alpha \cdot f_{c}^{b}
$$

Donde $a$ y $b$ son constantes.

Esta relación, para el hormigón objeto de estudio, se determinó por medio del complemento de Microsoft Excel - Solver. La condición de entrada fue minimizar el error.

La ecuación resultante que relaciona la resistencia a tracción y la resistencia a compresión viene dada por la expresión:

$$
f_{c t}=0,243 \cdot f_{c}^{0,67}
$$

Relacionando las ecuaciones [7] y [9] se obtiene que la resistencia de descimbrado para esta placa es de $f_{c, j}=10,85 \mathrm{MPa}$

\subsection{Determinación de la edad equivalente del elemento}

En la Figura 5 se muestra el perfil térmico para cada uno de los siete puntos instrumentados.

Con el historial de temperaturas de cada punto instrumentado, se determinó para cada uno la evolución del índice de madurez en el tiempo (véase en la Figura 6).

De la Figura 6 se concluye que instrumentando en diferentes puntos del elemento y a diferentes profundidades, existe variación considerable entre las edades equivalentes. Los puntos instrumentados en la mitad de la profundidad presentan una evolución de edad equivalente ligeramente más rápida que los puntos instrumentados a otras profundidades.
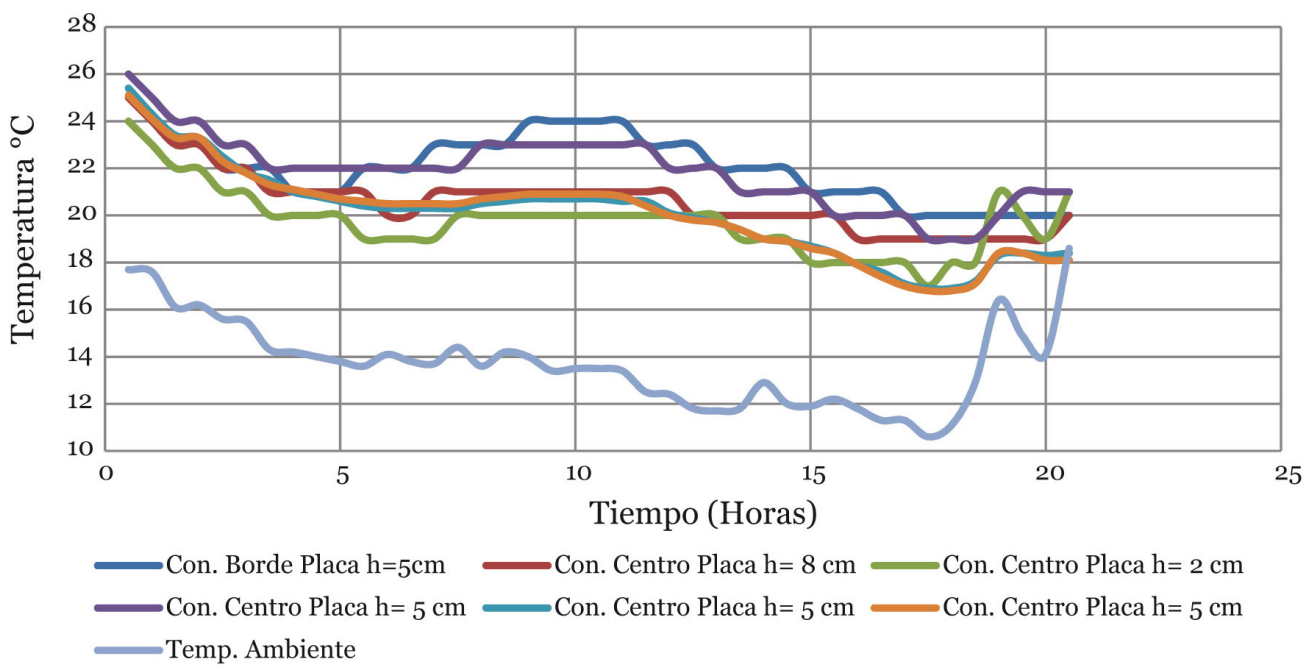

Figura 5. Historial de temperatura para diferentes puntos en la placa

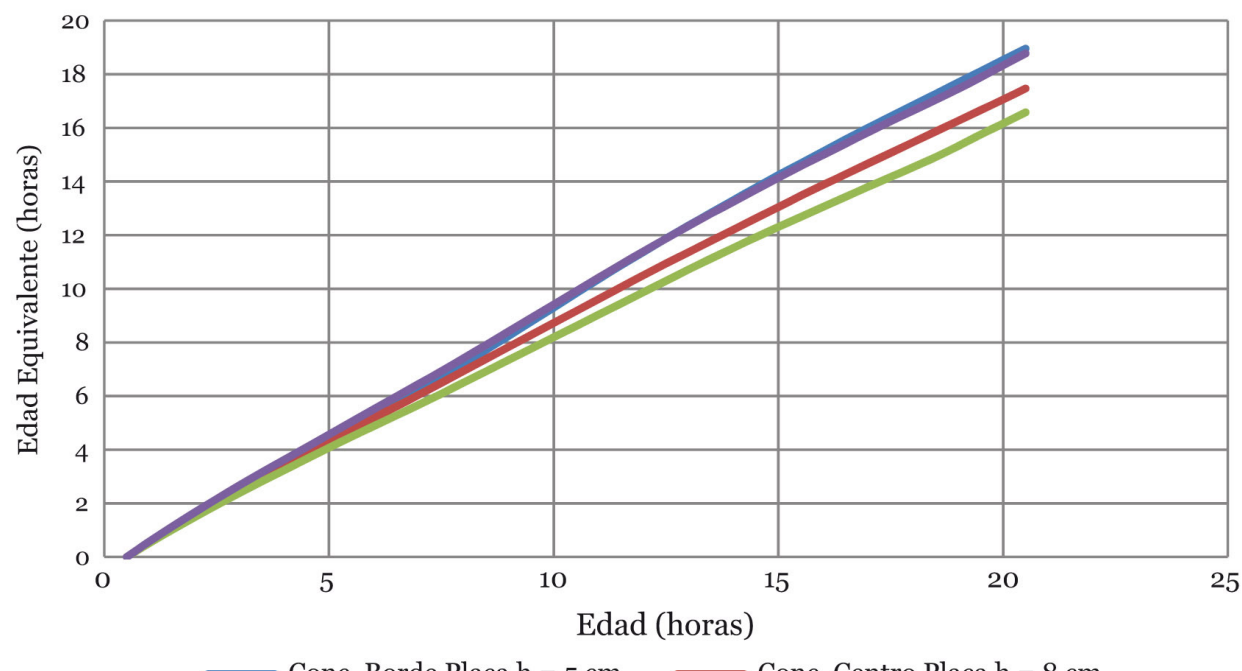

Figura 6. Comparación de Edades Equivalentes en los puntos instrumentados. 


\subsection{Determinación de tiempos de fraguado}

Se determinaron los tiempos de fraguado para cada punto instrumentado en la placa. Se determinó la edad equivalente para los tiempos inicial y final obtenidos en laboratorio, esto se muestra en la Figura 7.

Con las edades equivalentes para cada punto medido en la placa, se determinó en qué tiempo se presenta el índice de madurez $\mathrm{T}_{\mathrm{e}}$ para el tiempo de fraguado inicial y final. Los resultados consolidados se muestran en la Tabla 8.

En la Tabla 8 se observa que el punto más crítico en cuanto a tiempo de fraguado final es el centro de la placa a $2 \mathrm{~cm}$ de profundidad.

\subsection{Determinación de edad de descimbrado}

Se determinó la edad de descimbrado para cada punto medido sobre el elemento. Conociendo la ecuación de madurez obtenida en el laboratorio [3], que relaciona el logaritmo en base diez con la resistencia, y calibrada con los cilindros en obra, se procedió a determinar la edad equivalente en la que el hormigón llega a la resistencia de 10,85 MPa determinada con el método de Calavera (9). Con esto se obtuvo una edad equivalente de $\mathrm{T}_{\mathrm{e}}=13,49 \mathrm{~h}$.

Teniendo la edad equivalente que debe tener el hormigón para llegar a la resistencia de descimbrado, se calculó, en cada punto de la placa, la edad que ésta debía tener en condiciones no controladas para tener la misma resistencia de descimbrado. Los resultados se muestran en la Tabla 9.

Los resultados obtenidos muestran que el punto más crítico en cuanto al descimbrado es el centro de la placa a una profundidad de $2 \mathrm{~cm}$.

Por último, se comparó el valor de la edad de tiempo de fraguado final, con la edad de descimbrado calculada por el método de Calavera (9). Se observó que en todos los puntos de la placa, la edad de descimbrado es mayor a la del tiempo de fraguado final, en el caso más crítico la diferencia es de 11 horas.

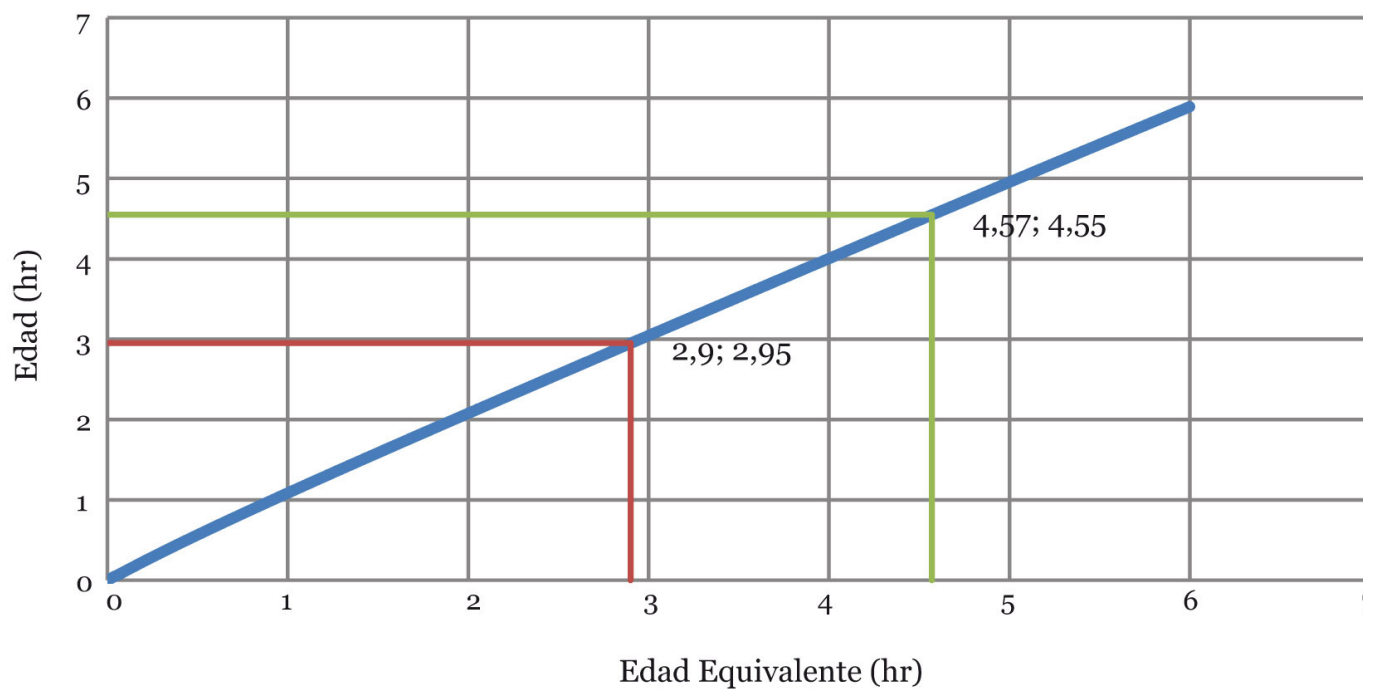

Figura 7. Determinación de edad equivalente para Tiempo de Fraguado inicial y final en placa.

Tabla 8. Tiempos de fraguado inicial y final para cada punto del elemento.

\begin{tabular}{|l|c|c|c|c|c|}
\cline { 2 - 6 } \multicolumn{1}{c|}{} & \multicolumn{4}{c|}{ Tiempos de Fraguado en placa } \\
\cline { 2 - 6 } \multicolumn{1}{c|}{} & $\mathbf{T}_{\mathbf{e}}(\mathbf{h})$ & $\begin{array}{c}\text { Borde Placa } \\
\mathbf{h}=\mathbf{5} \mathbf{c m}\end{array}$ & $\begin{array}{c}\text { Centro Placa } \\
\mathbf{h}=\mathbf{8 c m}\end{array}$ & $\begin{array}{c}\text { Centro Placa } \\
\mathbf{h}=\mathbf{2 c m}\end{array}$ & $\begin{array}{c}\text { Centro Placa } \\
\mathbf{h}=\mathbf{5} \mathbf{c m}\end{array}$ \\
\hline Inicial & 2,90 & 3,40 & 3,41 & 3,60 & 3,22 \\
\hline Final & 4,57 & 5,26 & 5,28 & 5,60 & 5,00 \\
\hline
\end{tabular}

Tabla 9. Edad de descimbrado para cada punto de la placa.

\begin{tabular}{|l|l|l|}
\hline \multicolumn{1}{|c|}{ Edad Descimbrado } & $\mathbf{T}_{\mathbf{e}}(\mathbf{h})$ & $\mathbf{t}(\mathbf{h})$ \\
\hline Borde Placa $\mathbf{h}=\mathbf{5} \mathbf{c m}$ & 13,49 & 14,0 \\
\hline Centro Placa $\mathbf{h}=\mathbf{8 c m}$ & 13,49 & 15,5 \\
\hline Centro Placa $\mathbf{h}=\mathbf{2 c m}$ & 13,49 & 16,5 \\
\hline Centro Placa $\mathbf{h}=\mathbf{5} \mathbf{c m}$ & 13,49 & 14,0 \\
\hline
\end{tabular}




\section{CONCLUSIONES}

La validación de la curva de madurez tanto para muro como para placa fue exitosa, con respecto al cálculo de la resistencia obtenida por el método de la madurez vs la resistencia determinada por medio de ensayos de resistencia a compresión de probetas cilíndricas en obra.

Una vez aplicado el método en la obra, se comprobó que a pesar que la placa tiene un espesor relativamente pequeño $(10 \mathrm{~cm})$, se presenta un diferencial térmico importante cuando la temperatura es medida en diferentes profundidades de la placa.

En el centro de la sección de la placa se presentan mayores temperaturas, lo cual representa una edad equivalente mayor. Por lo tanto, se puede inferir que el punto más crítico para la medición de la temperatura es en una profundidad de $2 \mathrm{~cm}$, por lo cual se recomienda instrumentar a esta profundidad al ser la más crítica.
La decisión de instrumentar a una profundidad de $5 \mathrm{~cm}$ o $2 \mathrm{~cm}$, representa una diferencia de dos horas y media en cuanto a la edad de descimbrado. Lo cual es crítico en sistemas industrializados.

Se comparó el valor de la edad de tiempo de fraguado final, con la edad de descimbrado calculada por el método de Calavera (9) y se observó que en todos los puntos de la placa, la edad de descimbrado es mayor a la del tiempo de fraguado final, en el caso más crítico la diferencia es de hasta 11 horas.

El tiempo de fraguado final no es un parámetro recomendable para descimbrar las placas, como lo recomiendan las productoras de hormigón. Por lo anterior, es importante verificar la edad a la que las placas alcanzan la resistencia para soportar las cargas de construcción.

\section{REFERENCIAS}

(1) Díaz, J.C., Bautista, L., Sánchez, A., Ruíz, D. (2004). Caracterización de mezclas de hormigón utilizadas en sistemas industrializados de construcción de edificaciones. Revista de ingeniería, 19: 60-73.

(2) Alvarado, Y.A., Calderón, P.A., Pallarés, F.J., Pellicer, T. (2006, 3-5 de Agosto). Estimation of shore removal times in bidirectional in situ concrete floor slabs applying the maturity method. En 1oth East Asia-Pacific conference on structural engineering and construction (pp. 55-60). Bangkok: Asian Institute of Technology.

(3) Alvarado, Y.A. (2009). Estudio experimental y numérico de la construcción de forjados hormigonados in situ mediante procesos de cimbrado, clareado y descimbrado de plantas consecutivas (Tesis Doctoral). Valencia: Universidad Politécnica de Valencia.

(4) Grundy, P., Kabaila, A. (1963). Construction loads on slabs with shored formwork in multistory buildings. ACI Journal Proceedings, 60(12): 1729-1738.

(5) Duan, M.Z., Chen, W.F. (1995). Improved simplified method for slab and shore load analysis during construction. (Project report CE-STR-95-24). West Lafayette Ind.: Purdue University.

(6) Fang, D-P., Geng, C-D., Zhu, H-Y., Liu, X-L. (2001). Floor load distribution in reinforced concrete buildings during construction. ACI Structural Journal, 98(2): 149-156, doi: http://dx.doi.org/10.14359/10182.

(7) Calderón, P. A., Alvarado, Y.A., Adam, J. M. (2011). A new simplified procedure to estimate loads on slabs and shoring during the construction of multistorey buildings. Engineering Structures, 33(5): 1565-1575, doi: http://dx.doi. org/10.1016/j.engstruct.2011.01.027.

(8) Castelli, N. (2012). Metodología de control de calidad de hormigones en obra, mediante calorimetría semi-adiabática de bajo costo (Proyecto de grado). Bogotá: Universidad de los Andes.

(9) Calavera, J., Fernández J. (1991). Cuaderno $N^{o}$ 3: Criterios para el descimbrado de estructuras de hormigón. Madrid: INTEMAC

(10) Lachemi, L., Hossain, K.M.A., Anagnostopoulos, C., Sabouni, A.R. (2007). Application of maturity method to slipforming operations: Performance validation. Cement \& Concrete Composites, 29(4): 290-299, doi: http://dx.doi.org/10.1016/j. cemconcomp.2006.12.001.

(11) ASTM International. (2008). ASTM C143. Standard Test Method for Slump of Hydraulic-Cement Concrete. American Society for Testing and Materials.

(12) ASTM International. (2008). ASTM C403. Standard Test Method for Time of Setting of Concrete Mixtures by Penetration Resistance. American Society for Testing and Materials.

(13) ASTM International. (2014). ASTM C39 Standard Test Method for Compressive Strength of Cylindrical Concrete Specimens. American Society for Testing and Materials.

(14) ASTM International. (2011). ASTM C496. Standard Test Method for Splitting Tensile Strength of Cylindrical Concrete Specimens. American Society for Testing and Materials.

(15) ASTM International. (2010). ASTM C78-10. Standard Test Method for Flexural Strength of Concrete (Using Simple Beam with Third-Point Loading). American Society for Testing and Materials.

(16) ASTM International. (2010). ASTM C469. Standard Test Method for Static Modulus of Elasticity and Poisson's Ratio of Concrete in Compression. American Society for Testing and Materials.

(17) ASTM International. (2011). ASTM C1074. Standard practice for estimating concrete strength by the maturity method. American Society for Testing and Materials.

(18) ASTM International. (2013). ASTM C109. Standard Test Method for Compressive Strength of Hydraulic Cement Mortars (Using 2-in. or [5O-mm] Cube Specimens). American Society for Testing and Materials.

(19) Castro-Garrido, M.C., López-Garzón, M.C. (2013). Aplicación del método de la madurez para la estimación de tiempos de fraguado y resistencias de hormigones industrializados con el fin de conocer la edad de descimbrado. (Proyecto de grado). Bogotá: Pontificia Universidad Javeriana. 
(20) Carino N J. The Maturity Method: Theory and Application (1984). Cement, Concrete, and Aggregates, 6(2): 61-73, doi: http://dx.doi.org/10.1520/CCA10358J

(21) Calavera, J. (2002). Cálculo, construcción, patología y rehabilitación de forjados de edificación. Madrid: INTEMAC.

(22) Ministerio de Ambiente, Vivienda y Desarrollo Territorial. (2010). Reglamento Colombiano de Construcción Sismo Resistente (NSR-1O). República de Colombia. 